\title{
JUSTIFICACIÓN DE LA VERTICALIDAD DE LOS TALUDES EN RAMBLAS DE CLIMA SEMIÁRIDO
}

\author{
Gonzalo Bianqui Mínguez \\ Margarita Box Amorós \\ Artemio Cuenca Payá
}

\section{INTRODUCCIÓN}

En los ríos y ramblas del sureste español, es regla general encontrar los lechos de avenida limitados por unos taludes verticales de material aluvial en el que se ha encajado el cauce, llegando a alcanzar alturas superiores a la veintena de metros y con una relación altura/anchura a veces superior a la unidad. Atendiendo a criterios geomorfológicos basados en análisis estadísticos de vertientes, podría pensarse que son formas elementales, poco evolucionadas ${ }^{1}$ pero, sin embargo, su asociación a redes altamente jerarquizadas ${ }^{2}$ obliga a buscar una explicación menos simplista.

Por otra parte, y desde la óptica de la Mecánica de Suelos, deberían ser taludes inestables dado que la fracción fina muy raramente supera el 50\% y está constituida, fundamentalmente, por limos con una mínima cantidad de arcilla, razón por la cual es presumible que el parámetro resistente básico venga determinado por el rozamiento interno.

Ahora bien, el hecho de que existan esos taludes verticales implica que hay una cohesión capaz de mantenerlos, y es a la estimación de la misma hacia

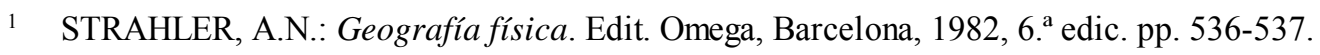

2 MORALES GIL, A., BRU RONDA, C. y BOX AMORÓS, M.: «Condiciones morfológicas y chubascos de fuerte intensidad horaria en la cuenca vertiente del Barranco de las Ovejas (Alicante)» en Lluvias torrenciales e inundaciones en Alicante. Serv. de Publicaciones de la Universidad de Alicante, Alicante, 1983. 
donde los autores han orientado el presente trabajo con el objetivo de determinar si es suficiente para justificar la estabilidad de esas formas.

\section{RESULTADOS}

Partiendo del criterio de rotura de Coulomb-Rankine ${ }^{3}$, la resistencia al corte de un suelo, viene dada por

$$
\mathrm{S}=\mathrm{c}+\mathrm{P}_{\mathrm{n}} \operatorname{tgn}
$$

en la que:

$\mathrm{s}=$ resistencia al corte

$\mathrm{c}=$ cohesión

$\mathrm{P}_{\mathrm{n}}$ fuerza normal al plano de corte

$\mathrm{n}=$ ángulo de rozamiento interno

El parámetro «c» puede tomarse como suma de dos componentes ${ }^{4}$

$$
\mathrm{C}=\mathrm{c}_{\mathrm{c}}+\mathrm{c}_{\mathrm{w}}
$$

en la que $\mathrm{c}_{\mathrm{c}}$ es la resistencia debida a enlaces rígidos formados por cementación y/o cristalización intergranular, $\mathrm{y}_{\mathrm{w}}$ la inducida por enlaces agua-suelo y por fuerzas capilares. La primera es muy importante en materiales arcillosos, especialmente con alto contenido en carbonato cálcico, mientras que la segunda domina en los suelos granulares (areno-limosos), por lo que la resistencia de un material de esta naturaleza queda condicionada, en gran parte, a su contenido en agua.

Para evaluar esa influencia, se han estudiado 31 muestras de material aluvial y coluvial de la comarca alicantina, tomadas entre 30 y $100 \mathrm{~cm}$ de profundidad, determinando la humedad natural y los límites de Atterberg, justificándose este último ensayo por el hecho de que los fenómenos de tensión superficial y de absorción se limitan a la fracción fina del suelo. Los resultados medios han sido los siguientes:

$\begin{array}{lrc} & \text { Media } & \text { Desviación standard } \\ \text { Límite líquido } & 26,8 & 3,7 \\ \text { Índice de Plasticidad } & 7,6 & 3,3 \\ \text { Humedad natural } & 4,7 & 3,2\end{array}$

3 Se ha estimado el criterio clásico de Coulom-Rankine por su facilidad de exposición y porque los resultados con él obtenidos difieren poco de los proporcionados por criterios más actuales y restrictivos, como los de Rowe y Skinner, al menos en cuanto al orden de valores que aquí se pretende determinar.

$4 \quad$ TSYTOVICH, N.: Soil Mechanics. Mir Publishers, Moscú, 1981, p. 57. 


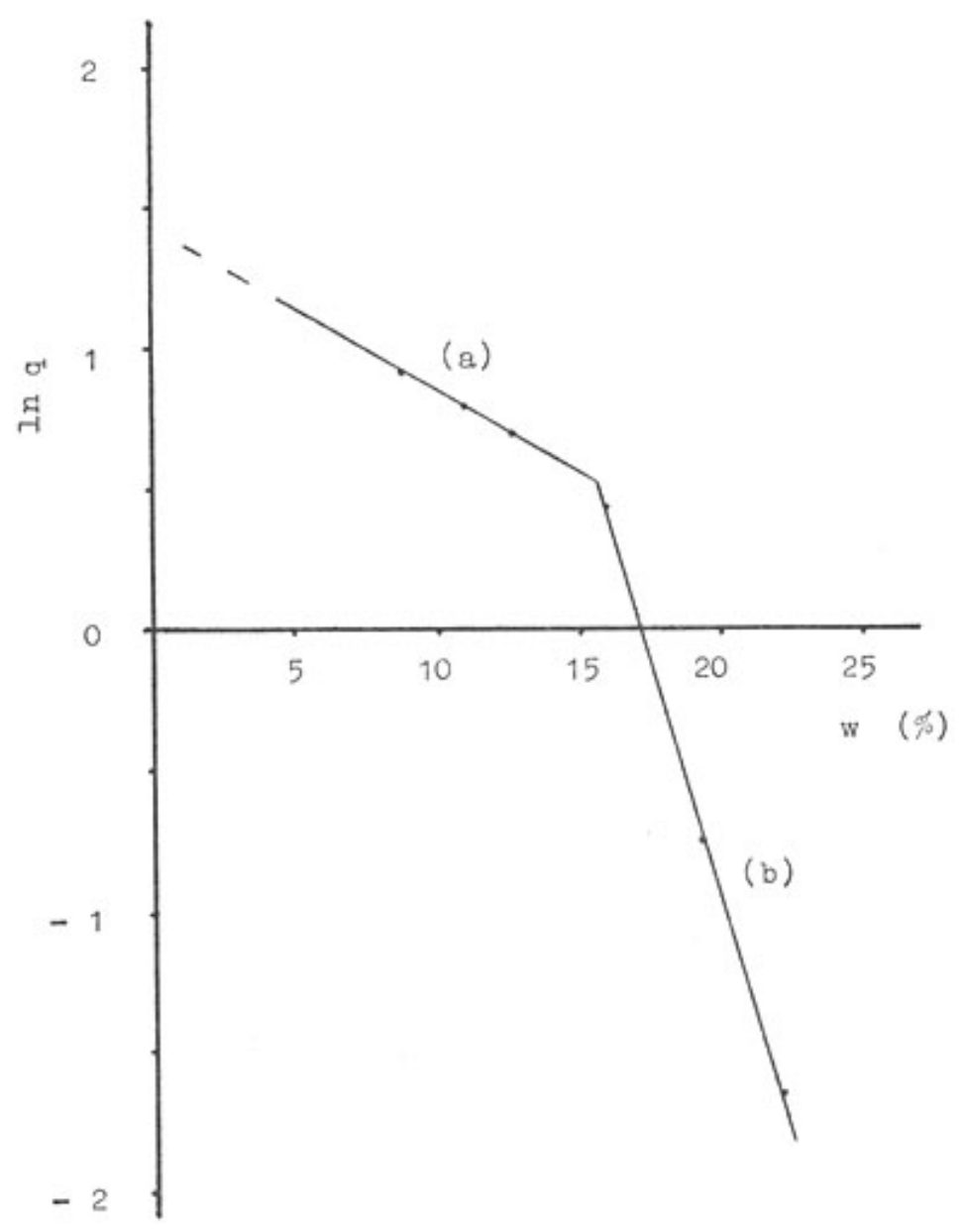

FIGURA 1. Disminución de la resistencia (q) con el incremento de la humedad (w)

Es notable la consistencia de resultados habida cuenta de que las muestras se tomaron a lo largo de casi treinta kilómetros, desde Alicante hacia el norte, y proceden de áreas litológicamente muy distintas. Asimismo, tampoco se apreció variación significativa en la humedad entre muestras tomadas de junio a agosto y otras tomadas en noviembre y diciembre.

Ante esto, se seleccionó un suelo con Límite Líquido 26,1 e Índice de Plasticidad 7,1 como suficientemente representativo, fabricando con él seis probetas (método Harvard miniatura) con distintas contenidos de humedad y rompiéndolas después de 24 horas en cámara húmeda a compresión simple, obteniéndose los datos expuestos en elCuadro I en el que 
$\mathrm{q}=$ resistencia a compresión simple $\left(\mathrm{Kg} / \mathrm{cm}^{2}\right)$

$\mathrm{w}=$ humedad

$\mathrm{e}=$ Indice de poros

\section{CUADRO I}

\begin{tabular}{|c|c|c|c|}
\hline Probeta & $q$ & $w$ & $e$ \\
\hline 1 & 2,49 & 8,8 & 0,67 \\
\hline 2 & 2,20 & 11,0 & 0,64 \\
\hline 3 & 1,99 & 12,8 & 0,57 \\
\hline 4 & 1,52 & 16,0 & 0,50 \\
\hline 5 & 0,47 & 19,2 & 0,57 \\
\hline 6 & 0,19 & 22,0 & 0,68 \\
\hline
\end{tabular}

La relación entre «q» $\mathrm{y}$ «W» se ha representado en escala semilogarítmica obteniéndose dos rectas (Fig. 1) de ecuaciones

$$
\begin{aligned}
& \ln q=1,409-0,056 w \\
& \ln q=5,951-0,347 w
\end{aligned}
$$

con unos coeficientes de correlación respectivos

$$
\begin{aligned}
& r_{a}=-0,999996 \\
& r_{b}=-0,999359
\end{aligned}
$$

El punto de intersección de estas rectas se encuentra en $\mathrm{w}=15,6 \%$ que es la humedad correspondiente a la denominada «óptima» en el ensayo de apisanado Harvard Miniatura para ese suelo, es decir, aquélla a partir de la cual la tensión superficial del agua intersticial deja de tener una influencia significativa y el material se aproxima al estado plástico. Respecto a los valores de «e», sólo se dispone de siete datos que oscilan entre un mínimo de 0,51 para el glacis aluvial de Campello y un máximo de 0,65 para la terraza inferior del Vinalopó en Elda, pero son suficientes para indicar el estado «denso» del material, característico de los sedimentos formados por corrientes con carga elevada y que es depositada rápidamente sin tiempo a una selección por tamaños.

Volviendo a las ecuaciones $(1, a)$ y $(1, b)$, puede apreciarse cómo existe una reducción de resistencia al aumentar la humedad, lenta hasta el punto de intersección y muy rápida a partir del mismo, confirmando el hecho de que en saturación, la resistencia debida a la cohesión prácticamente desaparece.

\section{DISCUSIÓN}

La homogeneidad en los resultados de humedad natural que no muestran variación entre los tomados en verano y los de invierno, es un reflejo del régi- 
men de precipitaciones, de forma que con totales medios anuales próximos a los $350 \mathrm{~mm}$, se caracteriza por aguaceros de fuerte intensidad horaria ${ }^{5}$, lo que da lugar a una importante escorrentía momentánea con un mínimo de infiltración, por lo que, exceptuando el entorno de la superficie mojada del cauce, no pueden esperarse variaciones importantes en el contenido de agua de la masa del suelo, hecho que permite tomar el valor medio encontrado e introducirlo en $(1, a)$ para estimar un valor de «q» del orden de $3,0 \mathrm{Kg} / \mathrm{cm}^{2}$. Tomando un valor medio de 1,7 $\mathrm{gr} / \mathrm{cm}^{3}$ para la densidad aparente del suelo, la altura crítica del talud vendrá dada por

$$
\mathrm{h}_{\mathrm{crit}}=\mathrm{n} \quad \frac{30}{1,7}
$$

en la que «n» es un coeficiente que vale 1,29 si la rotura se produce con formación de grieta en la coronación y 2,0 en caso contrario ${ }^{6}$. Se tiene, por tanto, que la altura crítica oscilará entre 35 y $23 \mathrm{~m}$, según el tipo de rotura y la profundidad de penetración de la grieta, caso de que se produzca ésta. Fórmulas con el mismo objetivo que la (2) han sido propuestas por numerosos autores con expresiones más complejas y separando el término de cohesión del de rozamiento interno, pero a efectos prácticos, los resultados son comparables, aunque algunos pueden llevar a engaño, pues la fórmula indicada en un conocido compendio de Geomorfología ${ }^{7}$ sobrevalora la altura crítica al no tener en cuenta el proceso de apertura de grietas.

Pasando ahora al caso de funcionamiento en el cauce por efecto de una avenida, el sector de talud situado debajo del nivel del agua, se irá saturando a medida que ésta penetre en su interior, llegando a superar el punto de intersección de las rectas $(1, a, b)$ con la consiguiente pérdida de resistencia y posterior colapso. Esto ocasionará la formación de una entalladura horizontal que descalzará el talud creando condiciones favorables para la rotura que se producirá según un plano vertical (Fig. 2), ya que es el que cumple la condición de mínima resistencia en función de los siguientes argumentos:

1) Para cualquier plano de corte inclinado un ángulo $\alpha$ respecto a la horizontal, la componente de resistencia por rozamientos es $\mathrm{P}$ sen (90- $\alpha) \operatorname{tg} \mathrm{n}$, siendo $\mathrm{P}$ el peso del macizo movilizado. Es elemental que con $\alpha=90^{\circ}$ ese término se anula.

2) Para ese mismo plano, simples consideraciones geométricas, permiten deducir que la resistencia debida a la cohesión $\left(\mathrm{F}_{\mathrm{c}}\right)$ es $\mathrm{F}_{\mathrm{c}}$, $=$ hc $\sec \left(90^{\circ}-\alpha\right)$, siendo h la altura del talud y c la cohesión. Nuevamente el mínimo de resistencia se obtiene con $\alpha=90^{\circ}$ para el cual $\mathrm{F}_{\mathrm{c}}=\mathrm{hc}$.

5 GIL OLCINA, A.: «Lluvias excepcionales en la noche del 19 al 20 de octubre de 1982 y riada en el Barranco de las Ovejas» en Lluvias torrenciales e inundaciones en Alicante. Op. cit.

6 TSCHEBOTARIOFF, G. P.: Mecánica del suelo. Aguilar, Madrid, 1963, pp. 169-170.

7 BRUNSDEN, D.: «Mass movements» en EMBLETON and THORNES, ed., Process in Goemorphology, Edward Ardnol Publishers, Londres, 1979, p. 136. 


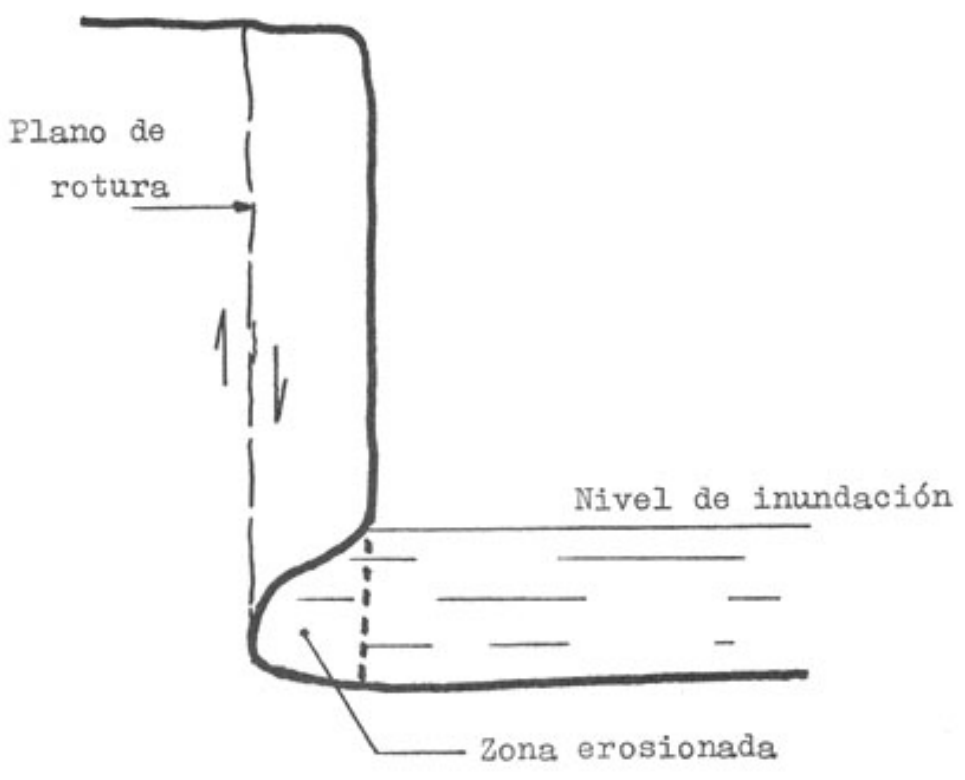

FIGURA 2. Esquema del proceso de regresión del talud

Se deduce, por tanto, que el retroceso del talud por efecto de la erosión en el pie se producirá manteniendo la misma morfología de pared vertical en tanto continúen las condiciones climáticas contrarias a procesos de infiltración profunda, capaces estos últimos, si se producen, de modificar el régimen de humedad en todo el perfil del talud merced a la reducción de las fuertes tensiones intersticiales negativas actualmente existentes.

\section{CONCLUSIONES}

De los estudios realizados, se deduce la existencia de una cohesión «aparente» debida a fenómenos de tensión superficial y de adsorción agua-suelo, suficiente para justificar la morfología geométricamente rectangular del perfil transversal de los cauces. Ésta tiene un doble origen climático basado en:

1) Los depósitos aluviales se han formado por sedimentación rápida de corrientes de elevada carga, características de los climas semiáridos, tal y como indican los valores del índice de poros.

2) La estabilidad de los taludes verticales, posteriormente excavados en ellos, es consecuencia de la baja pluviometría y de su distribución irregular que frena la infiltración, permitiendo la conservación de una humedad natural muy constante y alejada del punto a partir del cual el suelo queda saturado. 
Además, la evolución actual viene marcada por procesos de regresión del talud asociados a momentos de crecidas, pero que mantienen la inclinación vertical dado que, por los mismos motivos climáticos, no existen presiones intersticiales capaces de estructurar superficies de corte que penetren en el interior del talud a ángulos menores de $90^{\circ}$. 